\title{
Solvable models of interacting $n$-particle systems on quantum graphs
}

\author{
Jens Bolte and George Garforth
}

\begin{abstract}
We introduce $n$-particle quantum graphs with singular two-particle interactions in such a way that eigenfunctions can be given in the form of a Bethe ansatz. We show that this leads to a secular equation characterising eigenvalues of the Hamiltonian that is based on a finite-dimensional determinant. These findings generalise previous results about two-particle quantum graphs.
\end{abstract}

\section{Introduction}

A quantum graph is metric graph equipped with a differential operator that typically is a self-adjoint realisation of the differential Laplacian. Spectral properties of quantum graphs recently attracted much attention (see, e.g., $\mathbf{\mathbf { E K K } ^ { + } \mathbf { 0 8 }}$, $\mathbf{B K 1 3 a}$ ). They often mimic corresponding properties of Laplacians on manifolds and have become widely used models in quantum chaos KS99b, GS06. The popularity of quantum graph models is partly due to the fact that eigenvalues of compact quantum graphs can be characterised in terms of a secular equation involving a finitedimensional determinant [KS99b. This leads to a Gutzwiller-like trace formula that is an identity rather than an asymptotic relation KS99b, KN05, BE09. Moreover, numerical calculations, with the aim of analysing the distribution of eigenvalues, can be performed very efficiently [KS99b.

More recently, many-particle quantum systems on graphs with singular interactions have been introduced $\mathbf{B K 1 3 c}, \mathbf{B K 1 3 b}$, primarily with the intention to study the existence or absence of Bose-Einstein condensation BK14, BK16. The latter mostly requires knowledge about low-lying eigenvalues, which can be gained without a secular equation. As the classical configuration space of $n \geq 2$ particles on a graph is $n$-dimensional, one would, in general, not expect to find a secular equation based on a finite-dimensional determinant, but rather an approximate scheme involving a truncated determinant as, e.g., developed in DS92. However, it is well known that particular many-particle quantum systems, such as the LiebLiniger gas on an interval or a circle [LL63, Gau71, are solvable by making use of an $n$-particle Bethe ansatz for the eigenfunctions [Bet31, which is a finite sum of plane waves. In such a case only finitely many unknown coefficients need to be calculated in order to determine an eigenfunction. Although intervals and circles can be viewed as very simple graphs, an important step to generalising the Bethe ansatz

2010 Mathematics Subject Classification. 81Q35. 
to quantum graphs was done in [CC07, where the punctured real line, i.e. a graph with one vertex and two infinite edges, was equipped with a singular two-particle interaction that was specially designed to make a Bethe ansatz possible. In BG17. we extended this construction to two particles on arbitrary compact graphs, with explicit constructions for equilateral star graphs as well as for tetrahedra. In all cases the Bethe ansatz led to a secular equation with a finite-dimensional determinant. We numerically calculated eigenvalues and analysed their distribution finding that, on the scale of the mean level spacing, eigenvalues appear to be uncorrelated. As such an observation would normally be attributed to an integrable classical limit BT77, we see it as a hint of an underlying quantum integrable field theory (as it is the case for the Lieb-Liniger model).

In what follows we generalise the approach to $n$-particle graphs. We maintain the singular two-particle interactions between any pairs of the $n$ particles and show that the Bethe ansatz can be extended to this situation. We then derive a secular equation, which is our main result.

\section{Preliminaries}

2.1. One-particle quantum graphs. We denote by $\Gamma$ a metric graph with finite vertex set $\mathcal{V}$ and finite edge set $\mathcal{E}$. Each edge $e_{j} \in \mathcal{E}$ is assigned a (finite) length $l_{j}$. The one particle Hilbert space is

$$
L^{2}(\Gamma)=\bigoplus_{j=1}^{|\mathcal{E}|} L^{2}\left(0, l_{j}\right),
$$

and the Laplacian acting on $\Psi=\left(\psi_{j}\right)_{j=1}^{|\mathcal{E}|} \in H^{2}(\Gamma)$, with the Sobolev space $H^{2}(\Gamma)$ defined in analogy to $L^{2}(\Gamma)$, is

$$
\left.-\Delta_{1} \Psi=\left(-\psi_{j}^{\prime \prime}\right)\right)_{j=1}^{|\mathcal{E}|} .
$$

Self-adjoint realisations of the Laplacian can be characterised in terms of boundary conditions at the vertices [KS99a. Let

$$
\begin{aligned}
& \Psi_{\mathrm{bv}}=\left(\psi_{1}(0), \ldots, \psi_{|\mathcal{E}|}(0), \psi_{1}\left(l_{1}\right), \ldots, \psi_{|\mathcal{E}|}\left(l_{|\mathcal{E}|}\right)\right), \\
& \Psi_{\mathrm{bv}}^{\prime}=\left(\psi_{1}^{\prime}(0), \ldots, \psi_{|\mathcal{E}|}^{\prime}(0),-\psi_{1}^{\prime}\left(l_{1}\right), \ldots,-\psi_{|\mathcal{E}|}^{\prime}\left(l_{|\mathcal{E}|}\right)\right),
\end{aligned}
$$

be the vectors of boundary values and let $A, B$ be complex $|\mathcal{E}| \times|\mathcal{E}|$-matrices, such that $A B^{*}$ is self-adjoint and $(A, B)$ has maximal rank. Then the boundary conditions

$$
A \Psi_{\mathrm{bv}}+B \Psi_{\mathrm{bv}}^{\prime}=0
$$

define a domain of a self-adjoint realisation of the Laplacian as a subspace of $H^{2}(\Gamma)$. Further details can be found in, e.g., KS99a, GS06, BK13a.

The secular equation for Laplace eigenvalues requires two unitary $2|\mathcal{E}| \times 2|\mathcal{E}|$ matrices. One is the vertex $S$-matrix

$$
S_{v}(k)=-(A+i k B)^{-1}(A-i k B),
$$

with $k \in \mathbb{R}$, and

$$
T(k ; \mathbf{l})=\left(\begin{array}{cc}
0 & e^{i k \mathbf{l}} \\
e^{i k \mathbf{l}} & 0
\end{array}\right),
$$


where

$$
e^{i k \mathbf{l}}=\operatorname{diag}\left(e^{i k l_{j}}\right)_{j=1}^{|\mathcal{E}|} .
$$

Then, $k^{2}>0$ is an eigenvalue of the Laplacian with boundary conditions (2.5), iff

$$
\operatorname{det}\left(\mathbb{I}-S_{v}(k) T(k ; \mathbf{l})\right)=0,
$$

see KS99b, KS06].

2.2. Weyl group. Before we proceed it is useful to define the symmetric group $S_{n}$ and the Weyl group $\mathcal{W}_{n}$, which we use to characterise the symmetries of exactly solvable $n$-particle systems.

Elements $Q$ of the symmetric group $S_{n}$ of order $n$ ! will be written in terms of generators $T_{1}, \ldots, T_{n-1}$ which satisfy the conditions

(1) $T_{i} T_{i}=I$

(2) $T_{i} T_{j}=T_{j} T_{i}$ for $|i-j|>1$;

(3) $T_{i} T_{i+1} T_{i}=T_{i+1} T_{i} T_{i+1}$.

The $n-1$ generators of $S_{n}$ act on wave numbers $\left(k_{1}, \ldots, k_{n}\right)$ according to

$$
T_{i}\left(k_{1}, \ldots, k_{n}\right) \equiv\left(k_{T_{i}(1)}, \ldots, k_{T_{i}(n)}\right)=\left(k_{1}, \ldots, k_{i-1}, k_{i+1}, k_{i}, k_{i+2}, \ldots, k_{n}\right) .
$$

Definition 2.1. The Weyl group $\mathcal{W}_{n}$ is defined as a semidirect product of $(\mathbb{Z} / 2 \mathbb{Z})^{n}$ with the symmetric group $S_{n}$,

$$
\mathcal{W}_{n}:=(\mathbb{Z} / 2 \mathbb{Z})^{n} \rtimes S_{n}
$$

Some useful and well-known properties of the Weyl group are the following:

Proposition 2.2. The Weyl group $\mathcal{W}_{n}$ has order $2^{n} n$ ! and is generated by the elements $T_{1}, \ldots, T_{n}, R_{1}$ that satisfy the conditions

(1) $R_{1} R_{1}=I$

(2) $T_{i} T_{i}=I$;

(3) $T_{i} T_{j}=T_{j} T_{i}$ for $|i-j|>1$;

(4) $T_{i} T_{i+1} T_{i}=T_{i+1} T_{i} T_{i+1}$;

(5) $R_{1} T_{1} R_{1} T_{1}=T_{1} R_{1} T_{1} R_{1}$;

(6) $R_{1} T_{i}=T_{i} R_{1}$ for $i>1$.

The $n$ generators of $\mathcal{W}_{n}$ act on wave numbers $\left(k_{1}, \ldots, k_{n}\right)$ according to (2.10) and

$$
R_{1}\left(k_{1}, \ldots, k_{n}\right)=\left(-k_{1}, k_{2}, \ldots, k_{n}\right) .
$$

Finally it will be useful to relate the Weyl groups $\mathcal{W}_{n}$ and $\mathcal{W}_{n-1}$. To this end, it is convenient to define elements

$$
R_{i}=T_{i-1} \ldots T_{1} R_{1} T_{1} \ldots T_{i-1}
$$

so that

$$
R_{i}\left(k_{1}, \ldots, k_{n}\right)=\left(k_{1}, \ldots, k_{i-1},-k_{i}, k_{i+1}, \ldots, k_{n}\right),
$$

and the cyclic permutation

$$
C_{n}=T_{n-1} T_{n-2} \ldots T_{1}
$$

so that

$$
C_{n}\left(k_{1}, k_{2}, \ldots, k_{n-1}, k_{n}\right)=\left(k_{n}, k_{1}, \ldots, k_{n-2}, k_{n-1}\right)
$$


where we note the relation

$$
R_{n}=C_{n} R_{1} C_{n}^{-1} .
$$

The Weyl group $\mathcal{W}_{n}$ can then be written in terms of $\mathcal{W}_{n-1}$ according to

$$
\mathcal{W}_{n}=\left\{C_{n}^{d}\left(R_{n}\right)^{j} X ; X \in \mathcal{W}_{n-1}, d=0, \ldots, n-1, j=0,1\right\} .
$$

To simplify the notation we denote the action of any $P \in \mathcal{W}_{n}$ in analogy to (2.10) as

$$
P\left(k_{1}, \ldots, k_{n}\right)=\left(k_{P(1)}, \ldots, k_{P(n)}\right) .
$$

2.3. Bosons in an interval. In order to establish some key concepts in the $n$ particle setting, we begin by presenting the model of $n \delta$-interacting bosons confined to an interval of length $l$ first solved by Gaudin Gau71. The problem is formulated as a search for solutions of the formal Schrödinger equation

$$
\left(-\Delta_{n}+2 \alpha \sum_{i \neq j} \delta\left(x_{i}-x_{j}\right)\right) \psi\left(x_{1}, \ldots, x_{n}\right)=E \psi\left(x_{1}, \ldots, x_{n}\right)
$$

with $\alpha \in \mathbb{R}$ and particle positions $x_{1}, \ldots, x_{n}$ on the half-line $\mathbb{R}_{+}=(0, \infty)$. Here the $n$-particle Laplacian acts according to

$$
-\Delta_{n} \psi=-\sum_{j=1}^{n} \frac{\partial^{2} \psi}{\partial x_{j}^{2}} .
$$

For a suitable choice of a domain, the Laplacian becomes self-adjoint and provides a rigorous realisation of the formal operator in (2.20). Imposing bosonic symmetry

$$
\psi\left(x_{1}, \ldots, x_{n}\right)=\psi\left(x_{Q(1)}, \ldots, x_{Q(n)}\right),
$$

for all $Q \in S_{n}$, this domain can be shown to consist of the set of $n-1$ jump conditions in the derivatives,

$$
\left.\left(\frac{\partial}{\partial x_{j+1}}-\frac{\partial}{\partial x_{j}}\right) \psi\right|_{x_{j+1}=x_{j}^{+}}=\left.\alpha \psi\right|_{x_{j+1}=x_{j}^{+}},
$$

for $j \in\{1, \ldots, n-1\}$, and the Dirichlet condition

$$
\left.\psi\right|_{x_{1}=0}=0 .
$$

Due to the bosonic symmetry, $\psi$ can be restricted to the subspace

$$
d^{I}=\left\{\left(x_{1}, \ldots, x_{n}\right) \in \mathbb{R}_{+}^{n} ; x_{1}<\cdots<x_{n}\right\} .
$$

By applying a permutation $Q \in S_{n}$, it is also defined in all subspaces

$$
d^{Q}=\left\{\left(x_{1}, \ldots, x_{n}\right) \in \mathbb{R}_{+}^{n} ; x_{Q(1)}<\cdots<x_{Q(n)}\right\},
$$

and thus in all of $\mathbb{R}_{+}^{n}$. In the two-particle setting, interactions between particles means $\mathbb{R}_{+}^{2}$ is naturally dissected, along the line $x_{1}=x_{2}$, into two subspaces $d^{I}$ and $d^{T_{1}}$. Here, in the $n$-particle setting, appropriate dissections result in $n$ ! subspaces labeled by elements $Q \in S_{n}$. Bosonic symmetry establishes equivalence between each of these subspaces so that we need only consider one.

The task is then to construct explicit Laplace eigenfunctions $\psi$ in $d^{I}$ which satisfy conditions (2.23) and (2.24). The Bethe ansatz method in this context is 
the assumption that the appropriate ansatz is the sum of possible constituent plane wave states,

$$
\psi\left(x_{1}, \ldots, x_{n}\right)=\sum_{P \in \mathcal{W}_{n}} \mathcal{A}^{P} e^{i\left(k_{P(1)} x_{1}+\cdots+k_{P(n)} x_{n}\right)} .
$$

A function of this form is an eigenfunction of the Laplacian with eigenvalue

$$
E=\sum_{j=1}^{n} k_{j}^{2}
$$

The $\delta$-type boundary conditions (2.23) which characterise interactions between particles, imply the relations

$$
\mathcal{A}^{P T_{i}}=s_{p}\left(k_{P(i)}-k_{P(i+1)}\right) \mathcal{A}^{P},
$$

for $i \in\{1, \ldots, n-1\}$, with

$$
s_{p}(k)=\frac{k-i \alpha}{k+i \alpha}
$$

for all $P \in \mathcal{W}_{n}$. The Dirichlet condition (2.24) at the end-point of the half line implies the relation

$$
\mathcal{A}^{P R}=-\mathcal{A}^{P}
$$

for all $P \in \mathcal{W}_{n}$. Exact solvability is then assured if relations (2.29) and (2.31) are compatible with the properties of $\mathcal{W}_{n}$ as prescribed in Proposition 2.2. This amounts only to the requirement $s_{p}(u) s_{p}(-u)=1$ which is easily verified.

Until this point, particle positions have been defined on the half-line $\mathbb{R}^{+}$. Of course, in order to restrict the particles to an interval of length $l$ we must impose the further Dirichlet condition at the point $x=l$. Since bosonic symmetry allows us to restrict our attention to the domain $d^{I} \subset \mathbb{R}_{+}^{n}$, the appropriate boundary condition is given by

$$
\psi\left(x_{1}, \ldots, x_{n-1}, l\right)=0
$$

which implies the relation

$$
\begin{aligned}
\mathcal{A}^{P} & =-e^{-2 i k_{P(n)} l} \mathcal{A}^{P R_{n}} \\
& =-e^{-2 i k_{P(n)} l} \mathcal{A}^{P C_{n} R_{1} C_{n}^{-1}},
\end{aligned}
$$

where, for the latter equality, we have used (2.17). Finally, applying (2.29), (2.31) and (2.33) successively, we arrive at the condition

$$
e^{-2 i k_{P(n)} l}=\prod_{i=1}^{n-1} s_{p}\left(k_{P(n)}+k_{P(i)}\right) s_{p}\left(k_{P(n)}-k_{P(i)}\right)
$$

for all $P \in \mathcal{W}_{n}$. We note here that the form of $s_{p}(k)$ is such that, if (2.34) is satisfied for some $P \in \mathcal{W}_{n}$ then it is necessarily satisfied for elements

$$
P T_{1}, \ldots, P T_{n-2}, P R_{1} \text { and } P R_{n}
$$

in $\mathcal{W}_{n}$ and thus for every

$$
P X \text { and } P R_{n} X
$$


in $\mathcal{W}_{n}$ with $X \in \mathcal{W}_{n-1}$. Using (2.18), we then have that the condition (2.34) need only be satisfied for elements

$$
P \in\left\{I, C_{n}, C_{n}^{2}, \ldots, C_{n}^{n-1}\right\} .
$$

This is equivalent to the $n$ quantisation conditions

$$
e^{-2 i k_{j} l}=\prod_{i \neq j} s_{p}\left(k_{j}+k_{i}\right) s_{p}\left(k_{j}-k_{i}\right),
$$

with $j \in\{1, \ldots, n\}$. Solutions $\left(k_{1}, \ldots, k_{n}\right) \neq(0, \ldots, 0)$, such that $0 \leq k_{1} \cdots \leq k_{n}$, then constitute energies (2.28).

\section{General graphs with $\tilde{\delta}$-interactions}

Now we have established how to construct exactly solvable $n$-particle systems on an interval, we would like to extend the approach to general graphs. It turns out that systems of $\delta$-interacting particles on graphs with more than a single edge, in general, are not compatibile with the Bethe ansatz method. In BG17, which itself is based on a construction in $\mathbf{C C 0 7}$, graphs were equipped with singular, nonlocal interactions, referred to as $\tilde{\delta}$-type, designed to make a Bethe ansatz possible. In what follows we extend this approach to $n$-particle quantum graphs. Defining an appropriate $n$-particle Bethe ansatz, we show exact solvability and calculate a quantisation condition, in the form of a collection of $n$ secular equations, which provide the exact spectra.

Let us begin by viewing the compact graph $\Gamma$ in what we call its star representation $\Gamma^{(s)}$ by cutting all edges of $\Gamma$ to produce $|\mathcal{V}|$ star graphs and extending all the edges of the star graphs to infinity. The appropriate $n$-particle Hilbert space on $\Gamma^{(s)}$ is then

$$
\mathcal{H}_{n}^{(s)}=\bigotimes_{i=1}^{n}\left(\bigoplus_{j=1}^{|\mathcal{E}|} L^{2}(0, \infty)\right) .
$$

We remark that here $\mathcal{E}$ is now the union of the edge sets of all infinite star graphs. Its order is twice the number of edges of the compact graph $\Gamma$. Vectors

$$
\Psi=\left(\psi_{j_{1} \ldots j_{n}}^{(s)}\right)_{j_{1}, \ldots, j_{n}=1}^{|\mathcal{E}|}
$$

in $\mathcal{H}_{n}^{(s)}$ are then lists of $n$-particle functions

$$
\psi_{j_{1} \ldots j_{n}}^{(s)}: D_{j_{1} \ldots j_{n}}^{(s)} \rightarrow \mathbb{C}
$$

in $L^{2}\left(D_{j_{1} \ldots j_{n}}^{(s)}\right)$ with infinite subdomains defined as

$$
D_{j_{1} \ldots j_{n}}^{(s)}=(0, \infty)^{n} .
$$

The total configuration space for $n$ particles on $\Gamma^{(s)}$ is the disjoint union

$$
D_{\Gamma}^{(s)}=\bigsqcup_{j_{1}, \ldots, j_{n}=1}^{|\mathcal{E}|} D_{j_{1} \ldots j_{n}}^{(s)}
$$

of these subdomains. The $n$-particle Hilbert space can then be written $\mathcal{H}_{n}^{(s)}=$ $L^{2}\left(D_{\Gamma}^{(s)}\right)$. 
In the two-particle setting (see [BG17), interactions take place along the diagonals $x_{1}=x_{2}$ of two-dimensional configuration spaces $D_{m n}^{(s)}$. In the $n$-particle setting, we wish to impose interactions at the boundaries of subdomains

$$
D_{j_{1} \ldots j_{n}}^{(s, Q)}=\left\{\left(x_{1}, \ldots, x_{n}\right) \in D_{j_{1} \ldots j_{n}}^{(s)} ; x_{Q(1)}<\cdots<x_{Q(n)}\right\},
$$

with $Q \in S_{n}$. The appropriate total dissected configuration space is then

$$
D_{\Gamma}^{(s, *)}=\bigsqcup_{j_{1}, \ldots, j_{n}=1}^{|\mathcal{E}|}\left(\bigsqcup_{Q \in S_{n}} D_{j_{1} \ldots j_{n}}^{(s, Q)}\right),
$$

with the total dissected two-particle Hilbert space $\mathcal{H}_{n}^{(s, *)}=L^{2}\left(D_{\Gamma}^{(s, *)}\right)$. Thus vectors

$$
\Psi=\left(\left(\psi_{j_{1} \ldots j_{n}}^{(s, Q)}\right)_{j_{1}, \ldots, j_{n}=1}^{|\mathcal{E}|}\right)_{Q \in S_{n}}
$$

in $\mathcal{H}_{n}^{(s, *)}$ are lists of square-integrable functions $\psi_{j_{1} \ldots j_{n}}^{(s, Q)}: D_{j_{1} \ldots j_{n}}^{(s, Q)} \rightarrow \mathbb{C}$. The corresponding Sobolev space $H^{2}\left(D_{\Gamma}^{(s, *)}\right)$ is the set of $\Psi \in \mathcal{H}_{n}^{(s, *)}$ consisting of functions $\psi_{j_{1} \ldots j_{n}}^{(s, Q)} \in H^{2}\left(D_{j_{1} \ldots j_{n}}^{(s, Q)}\right)$.

Boundary conditions will be imposed on eigenfunctions $\Psi \in H^{2}\left(D_{\Gamma}^{(s, *)}\right)$ of the $n$-particle Laplacian $-\Delta_{n}$. We reiterate here that these will be $n$-particle analogues of the boundary conditions imposed in the two-particle setting in BG17. Before we proceed with establishing these conditions, it is convenient to define the permutation matrices $\mathbb{Q}$ as representations of $Q \in S_{n}$ on

$$
\bigotimes_{j=1}^{n} \mathbb{C}^{|\mathcal{E}|}
$$

such that

(1) $\mathbb{I}=\mathbb{I}_{|\mathcal{E}|^{n}}$ is the representation of $I$;

(2) $\mathbb{T}^{(i)}=\mathbb{I}_{|\mathcal{E}|^{i-1}} \otimes \mathbb{T}_{|\mathcal{E}|^{2}} \otimes \mathbb{I}_{|\mathcal{E}|^{n-i-1}}$ is the representation of $T_{i}$.

Here

$$
\mathbb{T}_{|\mathcal{E}|^{2}}=\left(\begin{array}{c}
\mathbb{I}_{|\mathcal{E}|} \otimes m_{1} \\
\vdots \\
\mathbb{I}_{|\mathcal{E}|} \otimes m_{|\mathcal{E}|}
\end{array}\right),
$$

with the $|\mathcal{E}|$-dimensional row vectors

$$
m_{j}=(0, \ldots, 0,1,0 \ldots, 0)
$$

in which the 1 is in $j$-th position. We note the properties

$$
\mathbb{T}^{(i)}\left(\mathcal{A}_{j_{1} \ldots j_{n}}\right)_{j_{1}, \ldots, j_{n}=1}^{|\mathcal{E}|}=\left(\mathcal{A}_{j_{1} \ldots j_{n}}\right)_{j_{1}, \ldots, j_{i-1}, j_{i+1}, j_{i}, j_{i+2}, \ldots, j_{n}=1}^{|\mathcal{E}|}
$$

for $|\mathcal{E}|^{n}$-dimensional column vectors $\mathcal{A}$ and that

$$
\begin{aligned}
& \mathbb{T}^{(i)}\left(M_{1} \otimes \cdots \otimes M_{i-1} \otimes M_{i} \otimes M_{i+1} \otimes M_{i+2} \otimes \cdots \otimes M_{n}\right) \mathbb{T}^{(i)} \\
& \quad=M_{1} \otimes \cdots \otimes M_{i-1} \otimes M_{i+1} \otimes M_{i} \otimes M_{i+2} \otimes \cdots \otimes M_{n}
\end{aligned}
$$

for any $|\mathcal{E}| \times|\mathcal{E}|$ matrices $M_{j}$. Finally, it is convenient to note the property

$$
\mathbb{Q}\left(M \otimes \mathbb{I}_{|\mathcal{E}|^{n-1}}\right) \mathbb{Q}^{-1}=\mathbb{I}_{|\mathcal{E}|} \otimes \cdots \otimes \mathbb{I}_{|\mathcal{E}|} \otimes M \otimes \mathbb{I}_{|\mathcal{E}|} \otimes \cdots \otimes \mathbb{I}_{|\mathcal{E}|}
$$

where on the right hand side, the matrix $M$ is the $Q(1)$-th position. 
Let us begin by establishing boundary conditions which prescribe single-particle interactions with the vertices. These will be given as simple $n$-particle lifts of those given in (2.5) imposed in the one-particle setting. The values of $\Psi \in H^{2}\left(D_{\Gamma}^{(s, *)}\right)$ at the vertices, along with corresponding derivatives, are given by boundary vectors

$$
\begin{aligned}
\Psi_{\mathrm{bv}}^{(v)} & =\left(\left(\left.\psi_{j_{1} \ldots j_{n}}^{Q}\left(x_{1}, \ldots, x_{n}\right)\right|_{x_{Q(1)}=0}\right)_{j_{1}, \ldots, j_{n}=1}^{|\mathcal{E}|}\right)_{Q \in S_{n}} ; \\
\Psi_{\mathrm{bv}}^{(v)^{\prime}} & =\left(\left(\left.\psi_{j_{1} \ldots j_{n}, Q(1)}^{Q}\left(x_{1}, \ldots, x_{n}\right)\right|_{x_{Q(1)}=0}\right)_{j_{1}, \ldots, j_{n}=1}^{|\mathcal{E}|}\right)_{Q \in S_{n}},
\end{aligned}
$$

where $\psi_{j_{1} \ldots j_{n}, Q(1)}^{Q}$ are inward derivatives normal to the lines $x_{Q(1)}=0$. Then, using (3.14), the appropriate boundary condition is given by

$$
\left(\mathbb{I}_{n !} \otimes \mathbb{Q}\left(A \otimes \mathbb{I}_{|\mathcal{E}|^{n-1}}\right) \mathbb{Q}^{-1}\right) \Psi_{\mathrm{bv}}^{(v)}+\left(\mathbb{I}_{n !} \otimes \mathbb{Q}\left(B \otimes \mathbb{I}_{|\mathcal{E}|^{n-1}}\right) \mathbb{Q}^{-1}\right) \Psi_{\mathrm{bv}}^{(v)^{\prime}}=0 .
$$

Here the matrices $A, B$ are of the same form as in (2.5) .

Next, we would like to impose $\tilde{\delta}$-type interactions between pairs of particles located on the same infinite star and impose continuity across dissections otherwise. Such interactions are prescribed in the $n$-particle setting according to the conditions

$$
\begin{aligned}
& \left.\psi_{j_{Q^{-1}(1)}^{Q} \cdots j_{Q^{-1}(n)}}^{Q}\left(x_{1}, \ldots, x_{n}\right)\right|_{x_{Q(i)}=x_{Q(i+1)}} \\
= & \left.\psi_{j_{T_{i} Q^{-1}(1)} \ldots j_{T_{i} Q^{-1}(n)}}\left(x_{1}, \ldots, x_{n}\right)\right|_{x_{Q(i)}=x_{Q(i+1)}} ; \\
& \left.\left(\frac{\partial}{\partial x_{Q(i+1)}}-\frac{\partial}{\partial x_{Q(i)}}-2 \alpha\right) \psi_{j_{Q^{-1}(1)} \ldots j_{Q^{-1}(n)}}^{Q}\left(x_{1}, \ldots, x_{n}\right)\right|_{x_{Q(i)}=x_{Q(i+1)}} \\
= & \left.\left(\frac{\partial}{\partial x_{Q(i+1)}}-\frac{\partial}{\partial x_{Q(i)}}\right) \psi_{j_{T_{i} Q^{-1}(1)} \ldots j_{T_{i} Q^{-1}(n)}}\left(x_{1}, \ldots, x_{n}\right)\right|_{x_{Q(i)}=x_{Q(i+1)}},
\end{aligned}
$$

if the edges $e_{j_{i}}$ and $e_{j_{i+1}}$ belong to the same star graph, and

$$
\begin{aligned}
& \left.\psi_{j_{Q^{-1}(1)} \cdots j_{Q^{-1}(n)}}^{Q}\left(x_{1}, \ldots, x_{n}\right)\right|_{x_{Q(i)}=x_{Q(i+1)}} \\
= & \left.\psi_{\left.j_{Q^{-1}(1) \cdots j_{Q^{-1}(n)}}\left(x_{1}, \ldots, x_{n}\right)\right|_{x_{Q(i)}=x_{Q(i+1)}} ;}\left(\frac{\partial}{\partial x_{Q(i+1)}}-\frac{\partial}{\partial x_{Q(i)}}\right) \psi_{j_{Q^{-1}(1)}^{Q} \cdots j_{Q^{-1}(n)}}\left(x_{1}, \ldots, x_{n}\right)\right|_{x_{Q(i)}=x_{Q(i+1)}} \\
= & \left.\left(\frac{\partial}{\partial x_{Q(i+1)}}-\frac{\partial}{\partial x_{Q(i)}}\right) \psi_{j_{Q^{-1}(1)} \cdots j_{Q^{-1}(n)}}\left(x_{1}, \ldots, x_{n}\right)\right|_{x_{Q(i)}=x_{Q(i+1)}},
\end{aligned}
$$

if the edges $e_{j_{i}}$ and $e_{j_{i+1}}$ belong to different star graphs.

The task is now to specify eigenvectors $\Psi \in H^{2}\left(D_{\Gamma}^{(s, *)}\right)$ which satisfy boundary conditions (3.16)-(3.18). Taking care to distinguish between subdomains $D_{j_{1} \ldots j_{n}}^{(s, Q)}$, the vector $\Psi$ will be described by the collection of functions

$$
\psi_{j_{1} \ldots j_{n}}^{Q}=\sum_{P \in \mathcal{W}_{n}} \mathcal{A}_{j_{1} \ldots j_{n}}^{(P, Q)} e^{i\left(k_{P(1)} x_{1}+\cdots+k_{P(n)} x_{n}\right)} .
$$

This form obviously leads to eigenfunctions of $-\Delta_{n}$ with Laplace eigenvalues (2.28).

Let us define the $|\mathcal{E}|^{n}$-dimensional vectors

$$
\mathcal{A}^{(P, Q)}=\left(\mathcal{A}_{j_{1} \ldots j_{n}}^{(P, Q)}\right)_{j_{1}, \ldots, j_{n}=1}^{|\mathcal{E}|}
$$


and then the $n !|\mathcal{E}|^{n}$-dimensional vectors

$$
\mathcal{A}^{P}=\left(\mathbb{Q}^{-1} \mathcal{A}^{\left(P Q^{-1}, Q\right)}\right)_{Q \in S_{n}} .
$$

It is convenient at this point to impose an ordering on (3.21) by associating with each element $Q$ the number $[Q] \in(1, \ldots, n !)$ so that

$$
\mathbb{Q}^{-1} \mathcal{A}^{\left(P Q^{-1}, Q\right)}
$$

is the $[Q]^{\text {th }}$ block in the list $\mathcal{A}^{P}$.

Boundary conditions at the vertices 3.16) imply the relations

$$
\mathbb{Q}^{-1} \mathcal{A}^{\left(P R_{Q(1)}, Q\right)}=\left(S_{v}\left(-k_{P Q(1)}\right) \otimes \mathbb{I}_{|\mathcal{E}|^{n-1}}\right) \mathbb{Q}^{-1} \mathcal{A}^{(P, Q)} .
$$

Noting then, that the properties of $\mathcal{W}_{n}$ imply

$$
R_{Q(1)}=Q R_{1} Q^{-1},
$$

we have that

$$
\mathcal{A}^{P R_{1}}=\mathbb{I}_{n !} \otimes S_{v}\left(-k_{P(1)}\right) \otimes \mathbb{I}_{|\mathcal{E}|^{n-1}} \mathcal{A}^{P} .
$$

At this point, it is convenient to define the diagonal matrices

$$
\boldsymbol{c}_{i}=\operatorname{diag}\left(c_{j_{1} \ldots j_{n}}^{(i)}\right)_{j_{1}, \ldots, j_{n}=1}^{|\mathcal{E}|},
$$

where $c_{j_{1} \ldots j_{n}}^{(i)}=1$, if the edges $e_{j_{i}}$ and $e_{j_{i+1}}$ belong to the same star graph, and $c_{j_{1} \ldots j_{n}}^{(i)}=0$ otherwise. These matrices distinguish domains with $\tilde{\delta}$-type interactions from those which are continuous across dissections. We notice here the relations

$$
\boldsymbol{c}_{i}=\mathbb{I}_{|\mathcal{E}|^{i-1}} \otimes \boldsymbol{c} \otimes \mathbb{I}_{|\mathcal{E}|^{n-i-1}},
$$

where $\boldsymbol{c}$ is defined for two-particle quantum graphs in BG17. The $\tilde{\delta}$-type conditions (3.17) and continuity conditions (3.18) imply the relations

$$
\begin{aligned}
& \left(\mathbb{I}_{2} \otimes \boldsymbol{c}_{i}\right)\left(\begin{array}{c}
\mathbb{Q}^{-1} \mathcal{A}^{\left(P T_{i} Q^{-1}, Q\right)} \\
\mathbb{T}^{(i)} \mathbb{Q}^{-1} \mathcal{A}^{\left(P Q^{-1}, Q T_{i}\right)}
\end{array}\right) \\
& \quad=\left(S_{p}\left(k_{P(i)}-k_{P(i+1)}\right) \otimes \mathbb{I}_{|\mathcal{E}|^{n}}\right)\left(\mathbb{I}_{2} \otimes \boldsymbol{c}_{i}\right)\left(\begin{array}{c}
\mathbb{Q}^{-1} \mathcal{A}^{\left(P Q^{-1}, Q\right)} \\
\mathbb{T}^{(i)} \mathbb{Q}^{-1} \mathcal{A}^{\left(P T_{i} Q^{-1}, Q T_{i}\right)}
\end{array}\right)
\end{aligned}
$$

and

$$
\left(\mathbb{I}_{|\mathcal{E}|^{n}}-\boldsymbol{c}_{i}\right) \mathbb{Q}^{-1} \mathcal{A}^{\left(P Q^{-1}, Q\right)}=\left(\mathbb{I}_{|\mathcal{E}|^{n}}-\boldsymbol{c}_{i}\right) \mathbb{Q}^{-1} \mathcal{A}^{\left(P Q^{-1}, Q T_{i}\right)}
$$

respectively. We then have that

$$
\mathcal{A}^{P T_{i}}=Y_{i}\left(k_{P(i)}-k_{P(i+1)}\right) \mathcal{A}^{P},
$$

where

$$
\left(Y_{i}(k)\right)_{[Q]\left[Q^{\prime}\right]}=\frac{-i \alpha}{k+i \alpha} \boldsymbol{c}_{i} \delta_{[Q]\left[Q^{\prime}\right]}+\left(\frac{k}{k+i \alpha} \boldsymbol{c}_{i}+\mathbb{T}^{(i)}\left(\mathbb{I}_{|\mathcal{E}|^{n}}-\boldsymbol{c}_{i}\right)\right) \delta_{\left[Q T_{i}\right]\left[Q^{\prime}\right]}
$$

Exact solvability is assured if relations (3.25) and (3.30) are compatible with the properties of $\mathcal{W}_{n}$. This amounts to the following consistency relations:

(1) $S_{v}(u) S_{v}(-u)=\mathbb{I}_{|\mathcal{E}|}$;

(2) $Y_{i}(u) Y_{i}(-u)=\mathbb{I}_{n !|\mathcal{E}|^{n}}$

(3) $Y_{i}(u) Y_{j}(v)=Y_{j}(v) Y_{i}(u)$ for $|i-j|>1$;

(4) $Y_{i+1}(u) Y_{i}(u+v) Y_{i+1}(v)=Y_{i}(v) Y_{i+1}(u+v) Y_{i}(u)$; 
(5) $\left(\mathbb{I}_{n !} \otimes S_{v}(u) \otimes \mathbb{I}_{|\mathcal{E}|^{n-1}}\right) Y_{1}(u+v)\left(\mathbb{I}_{n !} \otimes S_{v}(v) \otimes \mathbb{I}_{|\mathcal{E}|^{n-1}}\right) Y_{1}(v-u)$ $=Y_{1}(v-u)\left(\mathbb{I}_{n !} \otimes S_{v}(v) \otimes \mathbb{I}_{|\mathcal{E}|^{n-1}}\right) Y_{1}(u+v)\left(\mathbb{I}_{n !} \otimes S_{v}(u) \otimes \mathbb{I}_{|\mathcal{E}|^{n-1}}\right) ;$

(6) $Y_{i}(u)\left(\mathbb{I}_{n !} \otimes S_{v}(v) \otimes \mathbb{I}_{|\mathcal{E}|^{n-1}}\right)=\left(\mathbb{I}_{n !} \otimes S_{v}(v) \otimes \mathbb{I}_{|\mathcal{E}|^{n-1}}\right) Y_{i}(u)$ for $i>1$. These conditions can be verified by the explicit forms of $S_{v}(k)$ and $Y_{i}(k)$.

In order to turn the eigenfunctions in the star representation into eigenfunctions on the compact graph, we must impose appropriate joining conditions. These are the $n$-particle analogues of the joining conditions established in BG17. and are given by

$$
\psi_{j_{1} \ldots j_{n}}^{Q}\left(x_{1}, \ldots, x_{n}\right)=\psi_{j_{1}^{\prime} \ldots j_{n}^{\prime}}^{Q}\left(x_{1}^{\prime}, \ldots, x_{n}^{\prime}\right)
$$

for all $Q \in S_{n}$, where

$$
\left(x_{Q(1)}^{\prime}, \ldots, x_{Q(n)}^{\prime}\right)=\left(x_{Q(1)}, \ldots, x_{Q(n-1)}, l_{j_{Q(n)}}-x_{Q(n)}\right)
$$

and

$$
\left(j_{Q(1)}^{\prime}, \ldots, j_{Q(n)}^{\prime}\right)=\left(j_{Q(1)}, \ldots, j_{Q(n-1)}, j_{Q(n)}+|\mathcal{E}|\right) .
$$

We then have

$$
\begin{aligned}
\mathcal{A}^{P} & =E\left(-k_{P(n)}\right) \mathcal{A}^{P R_{n}} \\
& =E\left(-k_{P(n)}\right) \mathcal{A}^{P C_{n} R_{1} C_{n}^{-1}},
\end{aligned}
$$

where

$$
E(k)=\mathbb{I}_{n !|\mathcal{E}|^{n-1}} \otimes\left(\begin{array}{ll}
0 & 1 \\
1 & 0
\end{array}\right) \otimes e^{i k l} .
$$

Applying (3.25), (3.30) and (3.35) successively we have that the relation

$$
Z\left(k_{P(1)}, \ldots, k_{P(n)}\right)=0,
$$

with

$$
\begin{array}{r}
Z\left(k_{1}, \ldots, k_{n}\right)=\operatorname{det}\left[\mathbb{I}_{n !|\mathcal{E}|^{n}}-E\left(k_{n}\right) Y_{n-1}\left(k_{n}-k_{n-1}\right) \ldots Y_{1}\left(k_{n}-k_{1}\right)\right. \\
\left.\left(\mathbb{I}_{n !} \otimes S_{v}\left(k_{n}\right) \otimes \mathbb{I}_{|\mathcal{E}|^{n-1}}\right) Y_{1}\left(k_{1}+k_{n}\right) \ldots Y_{n-1}\left(k_{n-1}+k_{n}\right)\right],
\end{array}
$$

is satisfied for all $P \in \mathcal{W}_{n}$. By using properties of determinants, it can be shown that the explicit forms of $Y_{i}(k), S_{v}(k)$ and $E(k)$ are such that if (3.37) is satisfied for some $P \in \mathcal{W}_{n}$, then it is necessarily satisfied for elements

$$
P T_{1}, \ldots, P T_{n-2}, R_{1} \text { and } R_{n}
$$

in $\mathcal{W}_{n}$ and thus for every

$$
P X \text { and } P R_{n} X
$$

in $\mathcal{W}_{n}$ with $X \in \mathcal{W}_{n-1}$. Using (2.18), we can state the main result of this section.

THEOREM 3.1. Non-zero eigenvalues of a self-adjoint n-particle Laplacian $-\Delta_{n}$ defined on $\Gamma$ with local vertex interactions specified through $A, B$ and $\tilde{\delta}$-type interactions between particles when they are located on neighbouring edges, are the values $E=k_{1}^{2}+\cdots+k_{n}^{2} \neq 0$ with multiplicity $m$, where $\left(k_{1}, \ldots, k_{n}\right)$, such that $0 \leq k_{1} \leq \cdots \leq k_{n}$, are solutions to the $n$ secular equations

$$
Z\left(k_{i_{1}}, \ldots, k_{i_{n}}\right)=0,
$$

for $\left(i_{1}, \ldots, i_{n}\right) \in\left\{C_{n}^{d}(1, \ldots, n)\right\}_{d=0}^{n-1}$, with multiplicity $m$. 


\section{Recovering specific results}

In this final section, by choosing particular parameters, we show how to recover established results from the general $n$-particle quantisation condition prescribed by Theorem 3.1

4.1. Non-interacting particles. Non-interacting systems are recovered by reestablishing continuity across all dissected domains. This is achieved by setting $\boldsymbol{c}_{i}=\mathbf{0}$ for all $i \in\{1, \ldots, n-1\}$. Matrices

$$
\left.Y_{i}(k)\right|_{c=0}
$$

are then composed of blocks

$$
\left(\left.Y_{i}(k)\right|_{c=0}\right)_{[Q]\left[Q^{\prime}\right]}=\mathbb{T}^{(i)} \delta_{\left[Q T_{i}\right]\left[Q^{\prime}\right]} .
$$

By substituting into (3.38) we recover the secular equation (2.9) for the one-particle quantum graph.

4.2. Two-particle graphs. Simply by choosing $n=2$, one immediately recovers the spectra of two-particle quantum graphs as established in BG17] (see Theorem 5.1 therein).

\section{References}

[BE09] J. Bolte and S. Endres, The trace formula for quantum graphs with general self-adjoint boundary conditions, Ann. H. Poincaré 10 (2009), 189-223.

[Bet31] H. Bethe, Zur Theorie der Metalle. i. Eigenwerte und Eigenfunktionen der linearen Atomkette (German) [On the theory of metals. i. Eigenvalues and eigenfunctions of a linear chain of atoms], Z. Phys. 71 (1931), 205226.

[BG17] J. Bolte and G. Garforth, Exactly solvable interacting two-particle quantum graphs, J. Phys. A: Math. Theor. (2017).

[BK13a] G. Berkolaiko and P. Kuchment, Introduction to quantum graphs, Mathematical Surveys and Monographs, vol. 186, AMS, 2013.

[BK13b] J. Bolte and J. Kerner, Quantum graphs with singular two-particle interactions, J. Phys. A: Math. Theor. 46 (2013), 045206.

[BK13c] Quantum graphs with two-particle contact interactions, J. Phys. A: Math. Theor. 46 (2013), 045207.

[BK14] , Many-particle quantum graphs and Bose-Einstein condensation, J. Math. Phys. 55 (2014), 061901.

[BK16] J. Bolte and J. Kerner, Instability of Bose-Einstein condensation into the one-particle ground state on quantum graphs under repulsive perturbations, J. Math. Phys. $\mathbf{5 7}$ (2016), no. 4, 043301, 9.

[BT77] M. V. Berry and M. Tabor, Level clustering in the regular spectrum, Proc. Roy. Soc. Ser. A 356 (1977), 375-394.

[CC07] V. Caudrelier and N. Crampé, Exact results for the one-dimensional many-body problem with contact interaction: including a tunable impurity, Rev. Math. Phys. 19 (2007), no. 4, 349-370.

[DS92] E. Doron and U. Smilansky, Semiclassical quantization of chaotic billiards: a scattering theory approach, Nonlinearity 5 (1992), no. 5, 1055-1084.

$\left[\mathrm{EKK}^{+} 08\right]$ P. Exner, J. P. Keating, P. Kuchment, T. Sunada, and A. Teplyaev (eds.), Analysis on graphs and its applications, Proc. Symp. Pure Math., vol. 77, AMS, 2008, Papers from the program held in Cambridge, January 8-June 29, 2007.

[Gau71] M. Gaudin, Boundary energy of a Bose gas in one dimension, Phys. Rev. A. 4 (1971), 386-394.

[GS06] S. Gnutzmann and U. Smilansky, Quantum graphs: Applications to quantum chaos and universal spectral statistics, Advances in Physics 55 (2006), 527-625.

[KN05] P. Kurasov and M. Nowaczyk, Inverse spectral problem for quantum graphs, J. Phys. A.: Math. Gen. 38 (2005), 49014915. 
[KS99a] V. Kostrykin and R. Schrader, Kirchhoff's rule for quantum wires, J. Phys. A: Math. Gen. 32 (1999), 595-630.

[KS99b] T. Kottos and U. Smilansky, Periodic orbit theory and spectral statistics for quantum graphs, Ann. Physics 274 (1999), no. 1, 76-124.

[KS06] V. Kostrykin and R. Schrader, Laplacians on metric graphs: Eigenvalues, resolvents and semigroups, Contemp. Math. 415 (2006), 201-225.

[LL63] E. H. Lieb and W. Liniger, Exact analysis of an interacting Bose gas. I. The general solution and the ground state, Phys. Rev. (2) 130 (1963), 1605-1616.

Department of Mathematics, Royal Holloway, University of London, Egham, TW20 OEX, UK

E-mail address: jens.bolte@rhul.ac.uk

Department of Mathematics, Royal Holloway, University of London, Egham, TW20 0EX, UK

E-mail address: George.Garforth.2012@live.rhul.ac.uk 